NBER WORKING PAPER SERIES

THE EFFECT OF PHARMACEUTICAL
UTILIZATION AND INNOVATION
ON HOSPITALIZATION AND MORTALITY

Frank R. Lichtenberg

Working Paper 5418

NATIONAL BUREAU OF ECONOMIC RESEARCH 1050 Massachusetts Avenue

Cambridge, MA 02138

January 1996

This research was supported by Pfizer, Inc. I am grateful to Ashoke Bhattacharjya and Alison Keith for helpful discussions. John Ameriks provided capable research assistance. I am responsible for any errors. This paper is part of NBER's research programs in Productivity and Health Economics. Any opinions expressed are those of the author and not those of the National Bureau of Economic Research.

() 1996 by Frank R. Lichtenberg. All rights reserved. Short sections of text, not to exceed two paragraphs, may be quoted without explicit permission provided that full credit, including $(\mathcal{O}$ notice, is given to the source. 


\title{
THE EFFECT OF PHARMACEUTICAL \\ UTILIZATION AND INNOVATION \\ ON HOSPITALIZATION AND MORTALITY
}

\begin{abstract}
This paper presents an econometric analysis of the effect of changes in the quantity and type of pharmaceuticals prescribed by physicians in outpatient visits on rates of hospitalization, surgical procedure, mortality, and related variables. It examines the statistical relationship across diseases between changes in outpatient pharmaceutical utilization and changes in inpatient care utilization and mortality during the period 1980-92. The estimates indicate that the number of hospital stays, bed-days, and surgical procedures declined most rapidly for those diagnoses with the greatest increase in the total number of drugs prescribed and the greatest change in the distribution of drugs, by molecule. The estimates imply that an increase of 100 prescriptions is associated with 1.48 fewer hospital admissions, 16.3 fewer hospital days, and 3.36 fewer inpatient surgical procedures. A $\$ 1$ increase in pharmaceutical expenditure is associated with a $\$ 3.65$ reduction in hospital care expenditure.
\end{abstract}

Frank R. Lichtenberg

Graduate School of Business

Columbia University 3022 Broadway, Mail Code 9120

New York, NY 10027 and NBER 
I. Introduction

Case studies of a number of specific drugs have shown that these drugs reduced the demand for hospital care, and, in some cases, mortality. Other case studies have indicated that government-imposed rationing of pharmaceuticals led to increased use of hospital care. While these studies are valuable, the extent to which their findings apply to pharmaceutical use in general is unclear. Moreover, these studies have yielded mixed results about (or have not addressed) the issue of whether the reduction in hospital cost was outweighed by the increase in pharmaceutical cost.

In this paper we perform an econometric analysis of the effect of changes in the quantity and type of pharmaceuticals prescribed by all kinds of physicians, to all kinds of patients throughout the U.S., on rates of hospitalization, surgical procedure, mortality, and related variables. Our unit of analysis is a (ICD9 2-digit) disease or diagnosis; we control for the presence of "fixed (diagnosis) effects" by analyzing growth rates of the variables. To perform the analysis, we first construct a database on diagnosis-level inputs and outcomes at two points in time (1980 and 1991 or 1992). This entails the linkage of eight large files produced by the National Center for Health Statistics; each file contains between 30 thousand and 2 million records.

Aggregate data indicate that in 1991, people were seeing a doctor and consuming medicines about as often as they were in 1980 , but they were living longer and spending less time in hospitals. Since ambulatory care, pharmaceuticals, and inpatient care are the three major health inputs, and increased longevity is presumably a desired outcome (or output) of health care, one might interpret this as an indication of productivity growth in the health care sector. Many studies of other sectors of the economy (e.g. manufacturing) have shown that technological progress--the development and diffusion of new and improved products and processes--is an important source 
of productivity growth. The objective of the following analysis is to investigate the hypothesis that pharmaceutical innovation (reflected in changes in the distribution of drugs prescribed to patients) contributes to productivity growth: it reduces the demand for hospitalization (which accounts for almost half of U.S. health care expenditure) without reducing (and perhaps even increasing) life expectancy. 1

In the next section we summarize evidence provided by some of the previous research on this topic. We begin to discuss our own research design in section III, which describes the data on utilization of specific drugs, by patient diagnosis and year. These data enable us to construct an index of pharmaceutical innovation, hence to determine the impacts of such innovation--changes in the distribution of chemical substances prescribed by physicians to treat diseases. (We also examine the impact of innovations in surgical techniques.) Linkage of the drug data to data on inpatient and outpatient care utilization and mortality, and summary statistics for all variables, are discussed in section IV. Issues of econometric specification are treated in section V. The empirical results are presented in section VI, and

1 To assess the impact of (non-pharmaceutical) innovation on economic outcomes (e.g., productivity growth) in other sectors of the economy (e.g. manufacturing), economists sometimes use a cross-industry research design: they compare the productivity growth of sectors experiencing rapid technological progress with those experiencing slow technological progress. (For example, Griliches and Lichtenberg (1984) examined the relationship between R\&Dintensity and productivity growth at the industry level.) Our analysis fits within this framework: diseases correspond to different "bads", just as industries correspond to different "goods", and bads are equivalent to negative goods. The hypothesis that innovation has a positive impact on the growth of output of goods (conditional on input growth), also implies that it should have a negative impact on the growth of output of bads. 
section VII contains a summary and conclusions.

II. Evidence from previous studies

A report by the Boston Consulting Group (1993) provided anecdotal evidence about several major drugs. The following are three of the report's main conclusions. (1) Operations for peptic ulcers decreased from 97,000 in 1977, when H2 antagonists were introduced, to 19,000 in 1987--a reduction of 80 percent. This saved $\$ 224$ million in annual medical costs. (2) Before antibiotics, the typical [tuberculosis] patient spent three to four years in a sanatarium, and had a 30 to 50 percent chance of dying. Today, treated with antibiotics, he or she is highly likely to recover in six to 12 months.

Antibiotics have saved over $\$ 10$ billion since 1947 . (3) Until the late 1950 s, schizophrenia accounted for the vast majority of patients in mental hospitals, and two out of three schizophrenic patients spent the major portion of their lives under sedation in these institutions. By the late $1980 \mathrm{~s}, 95$ percent of patients were being treated on an outpatient basis. With the introduction of Clozapine in the late $1980 \mathrm{~s}$, many schizophrenic patients were able to return to work or undertake productive work for the first time. Drug treatments have saved the cost of keeping about 400,000 patients in mental institutions: about $\$ 25$ billion annually.

There have probably been many controlled studies of the impact of specific drugs. One recent study was the Scandinavian Simvastatin Survival Study of 4444 volunteers. The study indicated that "giving the drug simvastatin to heart patients reduced their hospital admissions by a third during five years of treatment. It also reduced the number of days that they had to spend in the hospital when they were admitted, and reduced the need for bypass surgery and angioplasty" (New York Times (1995a)). But treatment with the $\$ 2 /$ day pill that lowered cholesterol did not actually save money: hospital costs were $\$ 8$ million lower among the 2221 volunteers who 
got the drug, but the medicine itself cost $\$ 11$ million. The total number of days patients had to spend in the hospital for heart problems was reduced 34 percent.

Another recent study was the West of Scotland Coronary Prevention Study of 6595 ostensibly healthy men aged 45 through 64 . The results indicated that the cholesterol-lowering drug "pravastatin reduces the risk of heart attack and death in a broad range of people, not just those with established heart disease, but also among those who are at risk for their first heart attack" "Over five years, those [healthy individuals] treated with the cholesterol-lowering drug pravastatin suffered 31 percent fewer nonfatal heart attacks and at least 28 percent fewer deaths from heart disease than a comparable group of men who received a placebo." "In previous studies, pravastatin had been shown to reduce the risk of heart attack by 62 percent in patients with high cholesterol who already heart disease" (New York Times (1995b)). But the medication is expensive: it costs about $\$ 800$ a year to treat each person.

On the other hand, the clot-dissolving drug T.P.A., "costs $\$ 2,000$ to administer to each stroke victim, but has the potential to save much more in long-term care for those who are helped" (New York Times (1995c)).

Soumerai et al (1991) analyzed the effect of limits imposed by the New Hampshire Medicaid program on the number of reimbursable medications that a patient can receive on rates of admission to nursing homes and hospitals. Imposition of the reimbursement cap resulted in a $35 \%$ decline in drug use and an approximate doubling of the rate of nursing home admissions among chronically ill elderly patients. There was also a small, but statistically insignificant, increase in the hospitalization rate during the period of the cap.

Soumerai et al (1994) examined the effects of a three-prescription monthly payment limit (cap) on the use of psychotropic drugs and acute 
mental health care by noninstitutionalized patients with schizophrenia. The cap resulted in immediate $15-49 \%$ reductions in the use of antipsychotic drugs, antidepressants and lithium, and anxiolytic and hypnotic drugs. It also resulted in coincident (43-57\%) increases of 1 to 2 visits per patient per month to community mental health centers, sharp increases in the use of emergency mental health services and partial hospitalization, but no change in the frequency of hospital admissions. The estimated average increase in mental health care costs per patient during the cap $(\$ 1530)$ exceeded the savings in drug costs to Medicaid by a factor of 17 .

Although the studies surveyed above have shown that the consumption of certain specific drugs on an outpatient basis have reduced the demand for inpatient care (hospitalization and surgery), or that limitations on access to some drugs increased the demand for inpatient care, these studies may not reveal the effect of pharmaceuticals in general on the demand for inpatient care, since the drugs used (or to which access was denied) are not necessarily a random sample of all pharmaceuticals. It is possible, for example, that the distribution of benefits of pharmaceuticals (including reduced need for hospitalization and surgery) is highly skewed to the right--a few drugs confer enormous benefits, but the majority confer modest benefits ${ }^{2}$--and that the specific drugs enumerated above tend to be concentrated in the upper tail of the benefit distribution.

III. Data on utilization of specific drugs, by patient diagnosis and year

[Note Num]2The pharmaceutical industry is among the most R\&D intensive industries, and it is well known that the returns to R\&D are highly skewed. Of course, even the set of all drugs ever taken by patients is a highly nonrandom sample of all drugs ever pursued in R\&D by pharmaceutical companies, since the former must be approved by the FDA (i.e., they must be found to be "safe and effective.") 
Our objective is to determine the "aggregate or average" effect of changes in the quantity and type of drugs prescribed by physicians in outpatient visits on the frequency of hospitalization, performance of surgical procedures, and mortality. Our strategy is to examine the statistical relationship across diseases between changes in outpatient pharmaceutical utilization and changes in inpatient care utilization and mortality during the period 1980-92.

To perform the analysis, we require data at the disease level at at least two (reasonably distant) points in time on the distribution of drugs prescribed to treat that disease, the number of hospital stays and surgical procedures associated with (or due to) that disease, and mortality data by disease. We obtained data on drugs prescribed by physicians in outpatient visits, by disease, from the 1980 and 1991 National Ambulatory Medical Care Survey (NAMCS) Drug Mentions files produced by the National Center for Health Statistics (NCHS). NAMCS is a random sample of approximately 30 to 50 thousand outpatient visits that provides information about patient diagnoses, drugs prescribed by the physician during the visit, and other information about both the patient and the doctor; government estimates of the aggregate number of patient visits (by diagnosis, doctor specialty, etc.) and drug mentions are based on these surveys. The Drug Mentions files provide detailed data on the drugs prescribed in the (roughly $60 \%$ of) office visits in which at least one drug is prescribed. (Unfortunately, although NCHS has conducted the NAMCS survey since at least the early 1970s, 1980 is the first year in which it produced a Drug Mentions file containing the data coded in the way that we require.)

Each record in the Drug Mentions file includes a code for the specific drug prescribed, codes for up to three diagnoses (4-digit codes from the International Classification of Diseases, 9 th revision, clinical 
modification), and a "drug weight": a weight for computing population estimates of drug mentions from the survey data. If the drug is a combination drug (about $20 \%$ of the drugs are), the record specifies codes for up to five individual substances comprising the combination drug. We treated a prescription for a combination drug consisting of, for example, three substances, as three prescriptions, one for each substance. Because there can be multiple diagnoses cited in a given record, we sometimes confront the problem of "allocating" the mention of a drug across diagnoses. We adopted the simple, feasible, approach of equal allocation of the drug mention across the several diagnoses. For example, if two diagnoses were cited and the drug weight was 10,000 , we replaced the mention of that drug by two mentions of the same drug, one for each diagnosis, each with a drug weight of 5000 ; this procedure does not change the population estimates of drug mentions, by drug type.

Having dealt with the issues of combination drugs and multiple diagnoses in these ways, we constructed population (weighted) estimates of the number of drug mentions, by diagnosis and specific drug. In order to have a reasonably large average number of drug mentions per diagnosis ${ }^{3}$, we use the two-digit disease classification. (Examples of 2-digit disease categories are "hypertensive disease" and "pneumonia and influenza".) Let $\mathrm{N}_{\mathrm{ijt}}$ represent the population estimate of the number of mentions of molecular entity (drug) $i$ associated with 2-digit diagnosis $j$ in year $t(t=1980,1991)$.

Data on $\mathrm{N}_{\mathrm{ijt}}$ enable us to construct (noisy) indicators of the change during 1980-91 in the quantity and composition of drugs prescribed, by diagnosis.

Let $\mathrm{N}_{. \mathrm{jt}}=\sum_{\mathrm{i}} \mathrm{N}_{\mathrm{ijt}}$ denote the total number of mentions of all drugs associated

${ }^{3}$ As discussed in greater detail below, the larger a population estimate is, the greater its relative precision (the lower its relative standard error) 
with diagnosis $\mathrm{j}$ in year $\mathrm{t}$, and $\mathrm{n}_{\mathrm{ijt}}=\mathrm{N}_{\mathrm{ijt}} / \mathrm{N}_{\mathrm{jt}}$ denote drug i's share in total drugs prescribed for diagnosis $\mathrm{j}$ in year $\mathrm{t}$. Then QUANTITY $=\left(\mathbf{N}_{\mathrm{j}, 91} /\right.$ $\mathrm{N}_{. \mathrm{j}, 80}$ ) represents the ratio of total quantity of drugs prescribed for diagnosis $\mathrm{j}$ in 1991 to the corresponding quantity in 1980.

The data enable us to characterize the degree of similarity (or dissimilarity) of disease j's 1980 and 1991 distributions of drug mentions, by molecule. We seek to measure "how different" the mix of drugs prescribed in different years to patients with a given diagnosis was. We constructed the following index of the degree of dissimilarity of drugs prescribed in 1980 and 1991 , or "novelty" of drugs prescribed in 1991, relative to those described in 1980:

NOVELTY $Y_{j}=1-$

$$
\left\{\left(\sum_{\mathrm{i}} \mathrm{n}_{\mathrm{ij}, 80} \mathrm{n}_{\mathrm{ij}, 91}\right) /\left[\left(\sum_{\mathrm{i}} \mathrm{n}_{\mathrm{ij}, 80}{ }^{2}\right)\left(\sum_{\mathrm{i}} \mathrm{n}_{\mathrm{ij}, 91}\right)^{2 / 2}\right\}\right.
$$

This index is one minus the cosine of the percentage distributions of diagnosis j's 1980 and 1991 drug mentions, by molecule. ${ }^{4}$ It is bounded between zero and one; a value of zero indicates no novelty, i.e. perfect similarity of the two distributions, and a value of one indicates complete novelty, i.e. zero similarity. 5

4 Jaffe (1986) used the cosine of firms' distributions of patents by patent class to measure their technological proximity.

5 We also explored two other indexes of drug novelty at the disease level. The first was a chi-square statistic for testing the null hypothesis of independence of the 1980 and 1991 distributions of drug mentions, by molecule. This turned out not to be very informative, however: its magnitude was almost always very large--indicating rejection of the null hypothesis of identical distributions-and was extremely highly correlated with sample size (the total number of mentions in the two years). 
Percentage distributions of 1980 and 1991 drug mentions for all diagnoses, by specific drug, for the top 20 drugs $^{6}$ (ranked by total number of mentions), are shown in Table 1. In 1980, the most frequently prescribed drug was hydrochlorothiazide, which received an estimated $3.54 \%$ of the 743 million drug mentions; by 1991 , this drug's share of total mentions had declined by half, to $1.75 \%$. Amoxicillin was the most frequently prescribed drug in 1991 ; its share of total mentions was $3.71 \%$, having risen from $1.50 \%$ in 1980 .

Of course, the distribution of drugs prescribed in a given year (and changes in the distribution between years) varies considerably across diagnoses--this is precisely the variation we wish to exploit to analyze the effects of pharmaceutical innovation. To illustrate this heterogeneity, Table 2 shows the 1980 and 1991 distributions of the top 20 drugs prescribed to patients with a specific (and relatively common) diagnosis: ischemic heart disease and diseases of pulmonary circulation (ICD9 code 41). Two out of the top 3 drugs on this list (nitroglycerin and isosorbide) do not appear in the list of top 20 drugs for all patients. Four drugs (diltiazem, nifedipine,

The second index was the fraction of 1991 drug mentions that were for drugs that had been approved by the Food and Drug Administration after a given year (e.g., 1975 or 1980); drugs approved in 1982, for example, could not have been prescribed by doctors in 1980. This index was constructed by matching the 1991 Drug Mentions file to a list of FDA drug approvals obtained from the FDA. Unfortunately, we were unable to determine the FDA approval dates for about half of the drugs cited in the Drug Mentions file. Moreover, since there may be long and variable lags in the diffusion of drugs to patients after their approval by the FDA, it is not clear which approval date cut-off one should choose in constructing this index.

6 Overall, there were 754 distinct drugs cited in the 1980 NAMCS file, and 787 drugs in the 1991 file. 
Table 1

Frequency of 1980 and 1991 drug mentions for all diagnoses, by specific drug: Top 20 drugs

\begin{tabular}{lll} 
& \multicolumn{2}{c}{ YEAR } \\
& 1991 & 1980 \\
$\begin{array}{l}\text { Total Mentions } \\
\text { \% of Total } \\
\text { Mentions in Year: }\end{array}$ & $891 \mathrm{M}$. & $743 \mathrm{M}$ \\
& & \\
AMOXICILLIN & 3.71 & 1.50 \\
ACETAMINOPHEN & 3.18 & 2.00 \\
HYDROCHLOROTHIAZ & 1.75 & 3.54 \\
ASPIRIN & 1.48 & 2.78 \\
ERYTHROMYCIN & 1.80 & 2.11 \\
PHENYLPROPANOLAM & 1.30 & 2.23 \\
PHENYLEPHRINE & 1.44 & 2.07 \\
CODEINE & 1.42 & 1.75 \\
ALCOHOL & 0.91 & 2.23 \\
DIGOXIN & 1.17 & 1.61 \\
FUROSEMIDE & 1.14 & 1.34 \\
PENICILLIN & 0.34 & 2.28 \\
PSEUDOEPHEDRINE & 0.82 & 1.65 \\
IBUPROFEN & 1.49 & 0.78 \\
GUAIFENESIN & 1.14 & 1.19 \\
CHLORPHENIRAMINE & 0.70 & 1.60 \\
TETRACYCLINE & 0.49 & 1.74 \\
HYDROCORTISONE & 0.89 & 1.17 \\
NEOMYCIN & 0.72 & 1.31 \\
PROPRANOLOL & 0.59 & 1.39
\end{tabular}


Table 2

Frequency of 1980 and 1991 drug mentions for ICD9 code 41

(ischemic heart disease and diseases of pulmonary circulation), by specific drug: Top 20 drugs

\begin{tabular}{|c|c|c|}
\hline & \multicolumn{2}{|c|}{ YEAR } \\
\hline & 1980 & 1991 \\
\hline Total Mentions & $25 \mathrm{M}$ & $23 \mathrm{M}$ \\
\hline \multirow{2}{*}{\multicolumn{3}{|c|}{$\begin{array}{l}\% \text { of Total } \\
\text { Mentions in Year: }\end{array}$}} \\
\hline & & \\
\hline NITROGL YCERIN & 9.78 & 9.16 \\
\hline DIGOXIN & 9.74 & 5.52 \\
\hline ISOSORBIDE & 8.79 & 3.20 \\
\hline PROPRANOLOL & 9.70 & 1.95 \\
\hline ASPIRIN & 2.55 & 6.72 \\
\hline HYDROCHLOROTHIAZ & 5.84 & 2.59 \\
\hline FUROSEMIDE & 4.53 & 3.81 \\
\hline DILTIAZEM & 0.00 & 6.39 \\
\hline POTASSIUM REPLAC & 2.43 & 2.14 \\
\hline NIFEDIPINE & 0.00 & 4.60 \\
\hline TRIAMTERENE & 2.52 & 1.22 \\
\hline DIPYRIDAMOLE & 0.88 & 2.29 \\
\hline WARFARIN & 1.69 & 1.19 \\
\hline INSULIN & 1.09 & 1.68 \\
\hline LOVASTATIN & 0.00 & 2.63 \\
\hline ATENOLOL & 0.00 & 2.45 \\
\hline QUINIDINE & 1.63 & 0.56 \\
\hline METOPROLOL & 0.69 & 1.57 \\
\hline DIAZEPAM & 1.49 & 0.39 \\
\hline METHYLDOPA & 1.59 & 0.20 \\
\hline
\end{tabular}


lovastatin, and atenolol) that were not prescribed at all in 1980--in some cases because they had not been approved by the FDA by that year--together accounted for over $14 \%$ of 1991 drug mentions for patients with this diagnosis. But the fact that aspirin's share of drug mentions increased $164 \%$, from $2.55 \%$ to $6.72 \%$, indicates that "old" drugs may also experience sharp increases in market share.

As eq. (1) indicates, NOVELTY is calculated from estimated proportions of patients with a given diagnosis in a given year taking each specific drug. These proportions are subject to sampling error: for example, the standard error of $\mathrm{n}_{\mathrm{ij}, 80}$ is proportional to $\left[\left(\mathrm{n}_{\mathrm{ij}, 80}\left(1-\mathrm{n}_{\mathrm{ij}, 80}\right)\right) / \mathrm{N}_{\mathrm{j}, 80}\right]^{1 / 2}$. The standard error of the proportion is inversely related to the size of the denominator of the proportion. This suggests that the expected value of NOVELTY under the null hypothesis of no change in the distribution of drugs is inversely related to the (average) number of drug mentions for the diagnosis. In other words, relatively uncommon diagnoses are likely to have higher values of NOVELTY than common diagnoses. To see why, suppose that there are only two drugs, $A$ and $B$, and that the true (population) probability of taking drug $A$ is $50 \%$ in both years: zero novelty in the population. If we draw samples of size $\mathrm{N}$ in a given year, the proportion of the sample taking drug A may differ from $50 \%$; the smaller the sample, the greater the likelihood of observing a sample proportion less than $40 \%$ or greater than $60 \%$, for example. Consequently even if the population distribution has not changed, the 1980 and 1991 sample proportions are likely to be more dissimilar, and the NOVELTY index higher, the smaller the sample. This inverse relationship is quite evident in our data: the correlation coefficient across diagnoses between the log of the NOVELTY index and the $\log$ of the average of the number of drug mentions in 1980 and 1991 (denoted AVG_MENT) is -.61 , which is highly significant. To make accurate inferences about the effect of pharmaceutical innovation from the NOVELTY 
index, it will therefore be essential to control for sample (diagnosis) size. We do this by defining an "adjusted novelty" index ADJ_NOV as the residual from the regression of $\log$ (NOVELTY) on $\log$ (AVG_MENT). The coefficient on ADJ_NOV will then capture the effect of deviations of NOVELTY from the value implied (or predicted) by the diagnosis size.

IV. Linking drug data to data on inpatient and outpatient care utilization and mortality

To analyze the relationship between changes in the pattern of drug utilization and changes in the utilization of other medical inputs and mortality, we computed disease-level aggregate statistics from six additional NCHS data sets: the NAMCS 1980 and 1991 patient files, the 1980 and 1992 National Hospital Discharge Survey (NHDS) files, and the 1980 and 1991 Vital Statistics-Mortality Detail files.

The NAMCS patient files provide estimates of the number of outpatient visits, by disease, as well as the frequency of ambulatory surgical procedures and the frequency of referrals.

The NHDS is a survey of discharge records in a random sample of short-stay hospitals. Each wave of the NHDS contains about 250,000 records, which is roughly a $1 / 2-1 \%$ sample of the $30-40$ million annual hospital discharges. Each discharge record indicates: (1) up to seven patient diagnoses $^{7}$; (2) the number of nights the patient spent in the

7 The percentage distribution of non-newborn patients, by number of diagnoses, is as follows: No. of diagnoses Percent 
hospital; (3) the number of surgical procedures performed; and (4) discharge status (in particular, whether the patient was discharged dead). Hence, we can estimate the number of hospital stays (discharges), nights (or days), inpatient surgical procedures, and hospital deaths, by diagnosis ${ }^{8}$, in both 1980 and 1992.

Just as the Drug Mentions files report the specific drugs prescribed by physicians during office visits, the NHDS files disclose the specific surgical procedures performed in the course of hospital stays. Hence it is possible to construct, and we have constructed, measures of the novelty of surgical procedures analogous to the measures of drug novelty described above. Thus we can investigate the behavior of two types of medical innovation: pharmaceutical innovation and surgical innovation. We expect that these two types of innovation would have opposite effects on the demand for hospitalization and surgery: the adoption of new surgical procedures is likely to stimulate hospital admissions, whereas the adoption of new drugs is likely to reduce them.

8 In the absence of a better alternative, we allocated these variables equally across listed diagnoses in a manner similar to the one used for allocating drug mentions.

9 In principle, one would expect the surgical novelty index to be negatively correlated across diagnoses with the average number of surgical procedures, for the same reason that the drug novelty index is negatively correlated with the average number of drug mentions. This is not evident in our data, however: the correlation between the logs of the two surgery variables is essentially zero (.04). In subsequent analysis we therefore don't control for the 
The last two files we use are the 1980 and 1991 Vital StatisticsMortality Detail files. Unlike the other datasets we use, these are complete censuses as opposed to surveys: they include records (from death certificates) of each of the approximately 2 million U.S. deaths per year. (Apparently slightly less than half of deaths occur in hospitals.) Each record indicates the underlying cause of death (diagnosis), and the age at death, so that we can obtain (sampling-error-free) data on the number of deaths and mean age at death, by disease.

In principle, it is possible to extend this database on health inputs and outputs, by disease and year, in several ways. The National Medical Expenditure Survey (and its predecessors) provides detailed data on pharmaceutical and inpatient and outpatient care expenditure, and the National Health Interview Survey provides data on various health indicators (work-loss days, restricted activity days, self-reported health status). But these are household surveys believed to be subject to far greater reporting error (particularly with regard to diagnosis) than the provider surveys described above. Full investigation of these data is a task for future research. Summary statistics from the ambulatory care survey, hospital discharge survey, and mortality detail files are presented in Table 3. (Diagnosis-level data on all of the variables used in the empirical analysis are presented in a Data Appendix available upon request to the author.) The number of doctor office visits increased at roughly the same rate as the U.S. population, so that per capita office visits remained essentially constant at about 2.6. Drug mentions per office visit decreased from 1.78 to 1.63 .10

average number of surgical procedures

10 Only about $63 \%$ of office visits are "drug visits"-visits in which at least one drug is prescribed. However two or more drugs are prescribed in almost half of drug visits. 
Table 3

Summary Statistics

I. Aggregates from NAMCS Patient files

Office visits (millions)

$\underline{1980}$

463.3

Drug mentions (millions)

822.9

13.4

32.5

Ambul. surg. procs. (millions)

46,081

$\underline{1991}$

551.9

898.9

19.6

36.8

Number of records in file$$
33,795
$$

II. Aggregates from NHDS files

Hospital stays (millions)

Hospital days (millions)

Inpatient surg. procs. (millions)

$\underline{1980}$

34.7

$\underline{1992}$

258.8

28.2

28.5

179.5

38.1

Hospital deaths (millions)

0.95

0.79

Number of records in file

223,785

274,273

III. Aggregates from Vital Statistics-Mortality Detail files

Total deaths (millions)

Mean age at death (years) $\underline{1980}$

1.99

67.74 $\underline{1991}$

2.17

69.96 
The reported number of referrals by physicians to other physicians increased more than twice as fast as the number of office visits; the "propensity to refer" patients increased from $2.6 \%$ to $3.3 \%$. The number of ambulatory surgical procedures performed fell slightly, so that procedures per visit declined about $20 \%$.

Data from the hospital discharge surveys indicate a $20 \%$ decline in the number of hospital admissions. The aggregate number of hospital beddays fell almost twice as much as the number of hospital admissions, indicating a decline in average length of stay from 7.5 days to 6.3 days. The number of inpatient surgical procedures performed, however, increased by almost a third: average number of procedures per stay increased from 0.81 to 1.34. In both years, about $2.4 \%$ of hospital stays ended with the death of the patient

The mortality detail statistics indicate that the fraction of deaths occuring in hospitals fell from about $50 \%$ in 1980 to $38 \%$ in 1991 . The crude mortality rate declined: the population increased more than the number of deaths. Mean age at death (mean completed duration of life) increased more than 2 years $(3.3 \%)$ over the 11 -year period, from 67.7 years to 70.0 years.

V. Econometric specification

Our primary objective is to examine the effect of changes (from 1980 to 1991) in the quantity and distribution of drugs prescribed on changes in the utilization of other medical inputs (especially inpatient care), and

Moreover, about $17 \%$ of these drugs are combination drugs; we follow the NCHS practice of treating the mention of a combination drug as mentions of each of its ingredients. 
changes in mortality. We will use the adjusted drug novelty index

ADJ_NOV to measure the change in the percentage distribution of drug mentions, by molecule. We will also account for the change in the percentage distribution of surgical procedures, by type, by including the surgical novelty index SURG NOV in our regression equations.

When examining the relationship between changes in drug utilization and changes in inpatient care utilization and mortality, it is essential to attempt to control for changes in the incidence of diseases in the population. If the number of people suffering from a particular disease is increasing especially rapidly, we would expect both the number of drug mentions and the number of hospital stays associated with that disease to rise faster than average: exogenous changes in disease incidence are likely to induce a positive correlation between drug growth and hospital admissions growth. We attempt to control for changes in disease incidence by including as a regressor the growth rate in the number of patients diagnosed with the disease by physicians in outpatient visits (calculated from the NAMCS patient files). Because drugs are prescribed in about $60 \%$ of office visits, the correlation across diagnoses between the growth of patients (or visits) and the growth rate of drug mentions is very high--about .80 . When the growth in visits is included in the regression, the coefficient on the growth in drug mentions essentially reveals the effect of changes in the number of drug mentions per person visiting the doctor with that diagnosis on the number of hospital admissions per person visiting the doctor with that diagnosis. To the extent that the growth in outpatient visits is an imperfect indicator of true changes in disease incidence, the coefficient on changes in drug quantity is likely to be biased upwards: we are less likely to observe a negative association between this variable and the growth in hospital stays, even if one really exists. In this respect, our test of the hypothesis that increases in pharmaceutical quantity (and perhaps novelty) reduce the demand for hospitalization would 
be a "strong test."

The type of model we will estimate is of the form

$\ln \mathrm{Y}_{\mathrm{j}, 91}-\ln \mathrm{Y}_{\mathrm{j}, 80}=\boldsymbol{\beta}_{0}+\boldsymbol{\beta}_{1} \ln$ QUANTITY $_{\mathrm{j}}+\boldsymbol{\beta}_{2} \ln$ ADJ_NOV $_{\mathrm{j}}+$

$\boldsymbol{\beta}_{3} \ln$ SURG_NOV $_{j}+\boldsymbol{\beta}_{4} \ln$ VISITS $_{j}+\epsilon_{j}$

where $Y$ is a variable indicated in Table 3, such as the number of hospital

stays or mean age at death; $\mathrm{j}$ denotes the 2-digit ICD9 diagnosis; QUANTITY

is the ratio of 1991 to 1980 drug mentions; ADJ_NOV is the adjusted index

of drug dissimilarity described above; SURG_NOV is the index of surgical procedure dissimilarity; VISITS is the ratio of 1980 and 1991 outpatient visits; and $\epsilon$ is a disturbance. If $Y$ is defined as the number of hospital stays, then the hypothesis that, ceteris paribus, higher pharmaceutical utilization and innovation reduced growth in the demand for hospital stays implies that $\boldsymbol{\beta}_{1}<0$ and $\boldsymbol{\beta}_{2}<0$. It is perhaps worth noting that when we estimate this equation, we are analyzing the relationship between deviations from means: we are determining whether diagnoses with above-average pharmaceutical innovation tended to exhibit above-average declines in hospital stays. Factors other than pharmaceutical innovation (e.g., changes in government and private health insurance reimbursement policies) may have affected the average or aggregate incidence of hospitalization. If these unmeasured determinants of hospitalization did not vary much across diagnoses (or, if they did, were uncorrelated with ADJ_NOV), we will obtain unbiased estimates of the parameters of eq. (2).

There is very good reason to expect the disturbances of eq. (2) to be heteroskedastic. As reported in the NHDS documentation, the relative standard error (RSE) of an estimated $Y$ value--the ratio of the standard error (SE) of the estimate to the (point) estimate (Y) itself--is inversely related to $\mathrm{Y}$; in particular, $\operatorname{RSE}(\mathrm{Y})=\mathrm{SE}(\mathrm{Y}) / \mathrm{Y}=[\mathrm{a}+(\mathrm{b} / \mathrm{Y})]^{1 / 2}$, where $\mathrm{a}$ and $\mathrm{b}$ are constants. This is also a reasonable approximation for $\mathrm{SE}(\ln \mathrm{Y})$, so that $\operatorname{var}(\ln \mathrm{Y})=[\mathrm{a}+(\mathrm{b} / \mathrm{Y})]$. Under the reasonable assumption that sampling 
errors in the two years are independent, $\operatorname{var}\left(\ln \mathrm{Y}_{\mathrm{j}, 91}-\ln \mathrm{Y}_{\mathrm{j}, 80}\right)=[2 \mathrm{a}+\mathbf{b}$ $\left.\left\{\left(1 / \mathrm{Y}_{\mathrm{j}, 80}\right)+\left(1 / \mathrm{Y}_{\mathrm{j}, 91}\right)\right\}\right]$ : the variance of the growth rate is inversely related to the size of the estimates in both years. The reliability of estimates of the growth rate of hospital stays and related variables is greater for common diagnoses than for uncommon ones. 11

One would therefore expect the estimated growth rates for less common diagnoses to be further away from the mean growth rate (in both directions). We investigated this by calculating the correlations between squared deviations from mean growth rates and variables of the form $\{(1 /$ $\left.\left.\mathrm{S}_{\mathrm{j}, 80}\right)+\left(1 / \mathrm{S}_{\mathrm{j}, 91}\right)\right\}$, where $\mathrm{S}$ is one of several alternative measures of diagnosis size (total number of deaths, surgical procedures, or hospital beddays). Define $y_{j}=\left(\ln Y_{j, 91}-\ln Y_{j, 80}\right), y=\operatorname{mean}\left(y_{j}\right), y_{j}^{\prime}=\left(y_{j}-y\right)^{2}$, and $s_{j}=\left\{\left(1 / s_{j, 80}\right)+\left(1 / s_{j, 91}\right)\right\}$. Correlations between $y_{j}{ }^{\prime}$ and $s_{j}$ for different definitions of $\mathrm{Y}$ and $\mathrm{S}$ are shown in the following table ${ }^{12}$.

Y

STAYS DAYS NSURG HDEATH DEATHS AGE

$\underline{\mathrm{S}}$

$\begin{array}{lllllll}M & 0.72994 & 0.82797 & 0.10908 & 0.09452 & 0.01933 & 0.53581\end{array}$

$\begin{array}{llllll}0.0001 & 0.0001 & 0.2800 & 0.3649 & 0.8486 & 0.0001\end{array}$

S $\quad \begin{array}{lllllll}0.51375 & 0.50927 & 0.03345 & -0.02436 & -0.0106 & 0.2963\end{array}$

$\begin{array}{llllll}0.0001 & 0.0001 & 0.7287 & 0.8070 & 0.9162 & 0.0028\end{array}$

$\begin{array}{lllllll}\text { D } & 0.59387 & 0.58516 & 0.06099 & -0.01200 & -0.02325 & 0.35240\end{array}$

$\begin{array}{llllll}0.0001 & 0.0001 & 0.5268 & 0.9042 & 0.8184 & 0.0003\end{array}$

$M=$ total number of deaths

11 The size distribution of 2-digit ICD9 diagnoses is essentially lognormal: the majority of cases is accounted for by a relatively small number of diagnoses.

12 P-values are shown below the correlation coefficients. 
$\mathbf{S}=$ number of hospital stays (discharges)

$\mathrm{D}=$ number of hospital days

Several of the variables--STAYS, DAYS, and AGE--clearly exhibit heteroskedasticity: their squared deviations from mean growth rates are strongly positively correlated with all three inverse measures of diagnosis size. For all of the variables, the measure of diagnosis size whose inverse has the largest correlation with the squared deviation (an indicator of variance) is the number of deaths. Presumably this is partly due to the fact that the number of deaths is based on a complete census (it is not subject to sampling error), whereas the number of hospital stays and days is based on a random sample of less than $1 \%$ of hospitalizations. Moreover, a single diagnosis is cited in the mortality records, whereas up to seven diagnoses are cited in the hospital discharge survey; our procedure of allocating stays, days, etc., equally across diagnoses undoubtedly results in errors of measurement in the frequency of these variables, by diagnosis.

Because the dependent variables of eq. (2) exhibit marked heteroskedasticity, and their variance is most strongly inversely related to the number of deaths reported in the mortality detail files, we will estimate eq. (2) using weighted least squares, with weights equal to $\left\{\left(1 /\right.\right.$ DEATHS $\left._{\mathbf{j}, 80}\right)+$ $\left(1 /\right.$ DEATHS $\left.\left._{\mathbf{j}, 91}\right)\right\}^{-1}$. Diagnoses that are reported to have caused a larger number of deaths will receive greater weight in analyzing the relationship, across diagnoses, between pharmaceutical utilization and innovation and changes in hospitalization and mortality

\section{Empirical results}

Weighted least-squares estimates of eq. (2) are presented in table 4 In column 1 the dependent variable is the growth in hospital stays. The estimates indicate that there is a strong inverse relationship between the 
Table 4

Weighted least-squares estimates of eq. (2) (t-statistics in parentheses)

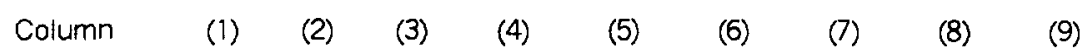

Dep. Var. STAYS DAYS ALOS NSURG HDEAT DEATH AGE AMBUL REFER

Regressor

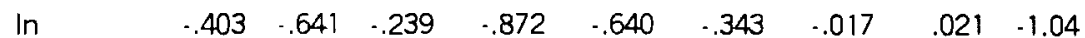

QUANTITY (2.91) $\left(\begin{array}{lllllllll}4.17) & (3.53) & (3.82) & (2.91) & (3.35) & (0.53) & (0.04) & (2.72)\end{array}\right.$

$\begin{array}{lllllllllll}\text { In } & -.215 & -.337 & -.122 & -.417 & -.423 & -.083 & -.005 & -.041 & -.137\end{array}$

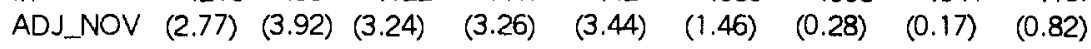

$\begin{array}{llllllllll}\text { In } & .269 & .280 & .010 & .553 & .143 & .107 & .016 & .069 & -.940\end{array}$

$\begin{array}{lllllllll}\text { SURG_NOV(3.40) } & (3.18) & (0.27) & (4.24) & (1.14) & (1.83) & (0.83) & (0.25) & (5.07)\end{array}$

$\begin{array}{llllllllll}\text { In VISITS } & .238 & .541 & .303 & .274 & .598 & .818 & .056 & .421 & .535\end{array}$

$\begin{array}{lllllllll}(1.30) & (2.67) & (3.39) & (0.91) & (2.06) & (6.07) & (1.28) & (0.64) & (1.20)\end{array}$

$\begin{array}{llllllllll}\text { Intercept } & .076 & -.252 & -.327 & 1.19 & -.374 & .148 & .049 & 1.04 & -.420\end{array}$

$\begin{array}{lllllllllll} & (1.07) & (3.22) & (9.52) & (10.3) & (3.35) & (2.84) & (2.91) & (4.51) & (2.67)\end{array}$

$\begin{array}{llllllllll}R^{2} & .274 & .332 & .187 & .436 & .187 & .384 & .040 & .023 & .317\end{array}$

$\begin{array}{llllllllll}\mathrm{N} & 93 & 93 & 93 & 93 & 93 & 93 & 93 & 74 & 74\end{array}$ 
growth in hospital stays and both the index of pharmaceutical innovation and the growth in total drug mentions: the number of hospital stays declined most rapidly for those diagnoses with the greatest increase in the total number of drugs prescribed and the greatest change in the distribution of drugs. The growth in stays is positively (and significantly) related to the extent of surgical innovation: the more dissimilar the 1980 and 1991 distributions of surgical procedures--presumably because of the adoption of new surgical techniques--the greater the increase in hospital admissions. About one-fourth of the cross-diagnosis variation in the growth of hospital stays is explained by the regressors.

The second column of Table 4 reports estimates of the regression of growth of total hospital bed-days on the same set of variables. The estimates are qualitatively similar to those in column 1 , but the magnitudes of the coefficients on In QUANTITY and ADJ_NOV are about 60\% larger. The growth rate of the average length of a hospital stay is equal to the growth rate of total hospital days minus the growth rate of the number of stays; the regression of this variable is shown in column 3 . These estimates suggest that greater quantity and novelty of pharmaceuticals had a negative impact on average length of stay in hospitals, as well as on the number of hospital stays. While diagnoses with high rates of surgical innovation had below-average declines in the number of hospital admissions, they had essentially average declines in average length of stay.

The dependent variable in column 4 is the growth in the number of inpatient surgical procedures performed. As in the total bed-days regression in column 2, the coefficients of both of the pharmaceutical variables are negative and highly significant, and the magnitude of the QUANTITY coefficient (as well as $\mathrm{R}^{2}$ ) is about 1/3 higher in column 4 . Increases in the quantity of pharmaceuticals prescribed appear to have a somewhat more negative impact on the growth of surgical procedures than they do on the 
grow th of hospital stays and total bed-days. This implies, of course, that the average number of procedures performed per stay increased more slowly for diagnoses with higher growth in drug quantity and novelty. The coefficient on SURG_NOV implies that diagnoses with greater surgical innovation exhibited higher growth in the average number of procedures per stay, as well as in the number of stays, which is not surprising.

Columns 5 through 7 present regressions of three different indicators of mortality: the number of deaths in hospitals, the total number of deaths, and mean age at death (for all deaths). The coefficients on drug quantity and novelty in the hospital deaths equation are similar to those in the DAYS equation, and larger than those in the STAYS equation. This implies that increases in these variables are associated with reductions in deaths per hospital stay (but not per hospital bed-day). The fact that the coefficient on the surgical innovation variable is much smaller in col. (5) than it is in col. (4) indicates that more rapid surgical innovation is associated with a decline in deaths per procedure. In the total deaths equation in col. (6), the coefficient on $\ln$ QUANTITY is again negative and significant--consistent with the hypothesis that increased pharmaceutical consumption reduces mortality rates--but it is only about half as large as its counterpart in col. (5), which implies that the nonhospital mortality rate is much less sensitive to pharmaceutical consumption than the hospital rate. The drug novelty variable has only a marginally significant negative effect on the increase in total deaths. The estimates in col. (7) reveal that none of the regressors have a significant impact on the change in completed life expectancy.

The first four regressions suggest that increases in drug consumption and novelty reduce the utilization of inpatient care; the next three regressions suggest that this does not come at the expense of higher mortality, and there is even some evidence that mortality is also reduced.

The last two regressions in Table 4 examine the behavior of two 
aspects of outpatient resource allocation: the number of ambulatory surgical procedures performed and the number of referrals to other physicians. In principle, it is possible that the reduction in inpatient surgical procedures associated with greater pharmaceutical utilization and novelty could be offset (partially or completely) by an increase in outpatient procedures; perhaps only the locus of performance of procedures changed (from hospital to doctor's office). The estimates in col. (8) do not support this conjecture: the change in ambulatory procedures appears to be unrelated to all of the regressors.

Estimates of the last equation reveal a significant negative correlation between the increase in the number of referrals (conditional on the number of office visits) and the increase in drug mentions (as well as the surgical novelty index). The greater the increase in the probability that the visited doctor prescribes a drug (or the expected number of drugs prescribed), the lower the increase in the probability that he or she refers the patient to another physician.

So far our discussion of empirical results has been concerned with hypothesis testing, i.e. with the signs and statistical significance of the parameter estimates reported in Table 4 . We now consider the magnitudes of the implied "marginal effects" of changes in the number of drug mentions on the dependent variables. In particular, we calculate the effect of an increase of 100 prescriptions on a variable $Y$ by multiplying the estimate of the elasticity $\beta_{1}$ by the ratio of aggregate $Y$ to aggregate drug mentions $M: d Y$ $/ \mathrm{dM}=\boldsymbol{\beta}_{1}\left(\mathrm{Y}_{\mathrm{A}} / \mathrm{M}_{\mathrm{A}}\right)$, where the $\mathrm{A}$ subscript denotes (average of 1980 and either 1991 or 1992) aggregate values. 13

\section{Dependent variable $\quad \underline{B}_{1} \quad \underline{Y}_{\mathrm{A}} \quad \underline{\mathrm{dY} / \mathrm{dM}=(\mathrm{Y}} \mathrm{A}-\underline{18.61)}$}

$13 \mathrm{Y}_{\mathrm{A}}$ is measured in millions. The mean of 1980 and 1991 aggregate drug mentions is 861 million. 


$\begin{array}{lccl}\text { STAYS } & -.403 & 31.6 & -1.48 \\ \text { DAYS } & -.641 & 219 & -16.3 \\ \text { NSURG } & -.872 & 33.2 & -3.36 \\ \text { HDEATH } & -.640 & .871 & -0.065 \\ \text { DEATHS } & -.343 & 2.08 & -0.083 \\ \text { REFER } & 1.04 & 16.5 & -1.99\end{array}$

The estimates imply that, holding constant the novelty of drugs and surgical procedures and the number of outpatient visits, an increase of 100 prescriptions is associated with 1.48 fewer hospital admissions, 16.3 fewer

hospital days, 3.36 fewer inpatient surgical procedures and 1.99 fewer outpatient referrals; there would be 83 fewer deaths ( 65 fewer hospital deaths) per 100,000 increase in prescriptions.

Using the following data on aggregate U.S. health expenditure in 1991 contained in the OECD Health Database, we can also attempt to estimate the effect of changes in pharmaceutical expenditure on inpatient care (and total health) expenditure:

Pharmaceutical expenditure: $\quad \$ 60.7 \mathrm{~b}$. Hospital care expenditure: $\quad 346.5 \mathrm{~b}$. Ambulatory care expenditure: $\quad 224.7 \mathrm{~b}$. --physicians' services expenditure $142.0 \mathrm{~b}$.

The parameter estimates imply that a $10 \%$ increase in drug mentions is associated with a $4.0 \%$ reduction in hospital stays and a $6.4 \%$ reduction in hospital beddays. We therefore think that it is reasonable to suppose that a $10 \%$ increase in pharmaceutical expenditure is associated with a $6.4 \%$ reduction in hospital care expenditure. ${ }^{14}$ (This estimate may be conservative because the surgery elasticity is larger in magnitude than the bed-days elasticity, and cost per bed-day is likely to increase with procedures per bed-

14 Total expenditure on hospital care presumably depends much more on the number of days than on the number of stays. 
day.) Since total expenditure on hospital care is 5.7 times as large as total pharmaceutical expenditure, this implies that a $\$ I$ increase in pharmaceutical expenditure is associated with a $\$ 3.65$ reduction in hospital care expenditure. This estimate implies that, if changes in pharmaceutical utilization had no other effects on health care costs, a $\$ 1$ increase in pharmaceutical expenditure would reduce total health care expenditure by $\$ 2.65$. But there are at least two reasons to believe that changes in pharmaceutical utilization would affect other costs, in both directions.

An increase in pharmaceutical utilization may necessitate an increase in ambulatory care utilization: a physician is required to prescribe the drugs. The slope coefficient from the (weighted) regression of the growth in office visits on the growth in drug mentions is .656: a $10 \%$ increase in drug mentions is associated with a $6.6 \%$ increase in office visits. If a $10 \%$ increase in drug expenditure would increase "expenditure on physicians" services" by $6.6 \%{ }^{15}$, a $\$ 1$ increase in drug expenditure would be associated with a $\$ 1.54$ increase in expenditure on physicians' services; this would offset $42 \%$ of the estimated reduction in inpatient expenditure. ${ }^{16}$

On the other hand, "hospital care expenditure" measures only the direct costs of hospitalization; it does not reflect the value of the patient's lost work and leisure time that presumably often accompanies hospitalization and surgery. If the indirect cost of hospitalization is, say, $25 \%$ as large as the

15 "Ambulatory care expenditure," as defined by OECD, also includes expenditure on dentists' services and on laboratory and diagnostic tests, which presumably need not increase with an increase in pharmaceutical utilization.

16 The fact that the probability of referral to another physician is inversely related to drug utilization suggests that these figures slightly overstate the increase in physicians' services associated with higher drug utilization. 
direct cost, then the reduction in the "social" (direct plus indirect)

hospitalization cost per dollar of increased pharmaceutical expenditure is $20 \%$

larger than the $\$ 3.65$ figure calculated above.

We conclude this section with a brief discussion of the magnitude of the coefficients on the pharmaceutical novelty index ADJ_NOV. The .25 and .75 quantiles of the ADJ_NOV distribution are -.113 and .288 , respectively. Thus the difference between the .75 and .25 quantile values is .402 , which is similar to the (unweighted) standard deviation of ADJ_NOV (.433). Below we calculate the predicted response of several dependent variables to a .402 increase in ADJ_NOV--a movement from the first to the third quartile of the adjusted novelty distribution--and compare it to the actual log change:

$$
\text { predicted change actual change }
$$

$\begin{array}{lcc}\text { STAYS } & -.086 & -.197 \\ \text { DAYS } & -.135 & -.369 \\ \text { SURG } & -.168 & +.301 \\ \text { HDEATH } & -.170 & -.182\end{array}$

The rate of growth of hospital stays of a diagnosis at the .75 percentile of the ADJ_NOV distribution is estimated to be 8.6 percentage points lower than that of a diagnosis at the .25 percentile.

By performing calculations similar to those made above with the QUANTITY coefficients, we could calculate the benefits (in the form of hospital cost reductions) of pharmaceutical novelty, i.e. of changes in the distribution of prescriptions, by drug. It is more difficult, however, to assess the cost of changing the distribution of drugs. R\&D expenditures by pharmaceutical firms represent a substantial part of these costs. According to the National Science Foundation, in 1991 these firms spent $\$ 6.1$ billion on R\&D. This is a very substantial amount, but it is only $1.8 \%$ of national expenditure on hospital care in that year. Thus pharmaceutical $R \& D$ 
spending would reduce total health expenditure (including pharmaceutical $R \& D)$ if it reduced hospital expenditure by as little as about $2 \%$. Our estimates indicate that changes in the distribution of pharmaceuticals are associated with significant hospital cost reductions. We hypothesize that the extent of changes in the pharmaceutical distribution are positively correlated across diagnoses with (lagged) R\&D expenditure, but since we lack data on R\&D spending by diagnosis 17 , we cannot examine the relationship between pharmaceutical R\&D and hospital costs directly.

\section{Summary and conclusions}

Case studies of a number of specific drugs have shown that these drugs reduced the demand for hospital care, and, in some cases, mortality. Other case studies have indicated that government-imposed rationing of pharmaceuticals led to increased use of hospital care. While these studies are valuable, the extent to which their findings apply to pharmaceutical use in general is unclear. Moreover, these studies have yielded mixed results about (or have not addressed) the issue of whether the reduction in hospital cost was outweighed by the increase in pharmaceutical cost.

In this paper we have examined the effect of changes in the quantity and type of pharmaceuticals prescribed by all kinds of physicians, to all kinds of patients throughout the U.S., on rates of hospitalization, surgical procedure, mortality, and related variables. Our unit of analysis was a (ICD9 2-digit) disease or diagnosis, which we argued is analagous to a product (or industry)

17 Cockburn and Henderson have compiled data on pharmaceutical R\&D at the "program" (and even the project) level. In principle, it might be possible to calculate $R \& D$ expenditure by diagnosis from their data, but unfortunately they are proprietary. 
in industrial organization economics. We controlled for the presence of "fixed (diagnosis) effects" by analyzing growth rates of the variables. To perform the analysis, we first constructed a database on diagnosis-level inputs and outcomes at two points in time (1980 and 1991 or 1992). This entailed the linkage of eight large files produced by the National Center for Health Statistics; each file contained between 30 thousand and 2 million records.

The limitations of the available data posed a number of statistical problems. The diagnosis-level data on drug utilization, ambulatory care, and hospitalization that we constructed are based on surveys, and are subject to sampling error. Moreover, these errors are heteroskedastic; we therefore estimated models using weighted least squares. Also, due to the presence of sampling error, the expected value of the drug novelty index is inversely related to sample size; we corrected for this by using an adjusted novelty index. Exogenous changes in disease incidence are likely to induce a ("spurious") correlation between drug growth and hospital admissions growth, although both theory and evidence suggest that this will bias the correlation upward, making our hypothesis tests "strong tests." We attempted to control for changes in disease incidence by including as a regressor the growth rate in the number of patients diagnosed with the disease by physicians in outpatient visits. An additional problem was posed by multiple diagnoses sometimes being cited in connection with a single drug mention, office visit, or hospital stay; lacking any alternative, we simply allocated the "event" equally across listed diagnoses.

Our principal findings may be summarized as follows. (1) The number of hospital stays, bed-days, and surgical procedures declined most rapidly for those diagnoses with the greatest increase in the total number of drugs prescribed and the greatest change in the distribution of drugs. The estimates imply that an increase of 100 prescriptions is associated with 1.48 fewer hospital admissions, 16.3 fewer hospital days, and 3.36 fewer inpatient 
surgical procedures. (2) Greater quantity and novelty of pharmaceuticals had a negative impact on average length of stay in hospitals, as well as on the number of hospital stays. (3) The average number of inpatient procedures performed per stay increased more slowly for diagnoses with higher growth in drug quantity and novelty. (4) Increases in drug quantity and novelty are associated with reductions in both the number of hospital deaths and deaths per hospital stay; they have much weaker effects on nonhospital mortality, and are unrelated to changes in mean age at death. (5) The greater the increase in the probability that a doctor prescribes a drug, the lower the increase in the probability that he or she refers the patient to another physician. (6) Changes in the number of ambulatory surgical procedures appear to be unrelated to changes in drug utilization. (7) A $\$ 1$ increase in pharmaceutical expenditure is associated with a $\$ 3.65$ reduction in hospital care expenditure (ignoring any indirect cost of hospitalization), but it is also associated with a $\$ 1.54$ increase in expenditure on ambulatory care. (8) Diagnoses subject to higher rates of surgical innovation exhibited larger increases (or smaller declines) in hospitalization, and marginally significantly larger increases in mortality. 


\section{REFERENCES}

Boston Consulting Group, Inc. (1993), "The Contribution of Pharmaceutical

Companies: What's at Stake for America, Executive Summary," September.

Griliches, Zvi, and Frank Lichtenberg (1984), "R\&D and Productivity at the Industry Level: Is There Still a Relationship?," in $\underline{R} \& D$, Patents, and

Productivity, Zvi Griliches, ed. (Chicago: Univ. of Chicago Press).

Jaffe, Adam (1986), "Technologivcal Opportunity and Spillovers of R\&D:

Evidence from Firms' Patents, Profits, and Market Value," American

Economic Review 76, December, pp. 984-1001.

New York Times (1995a), "Cholesterol pill linked to lower hospital bills,"

March 27, p. All.

New York Times (1995b), "Benefit to healthy men is seen from cholesterolcutting drug," November 16, p. Al.

New York Times (1995c), "New study finds treatment helps stroke patients,"

December 14, Al

Soumerai, Stephen, et al (1991), "Effects of Medicaid drug-payment limits on admission to hospitals and nursing homes," The New England Journal of Medicine 325 (15), October 10, pp. 1072-7.

Soumerai, Stephen, et al (1994), "Effects of limiting Medicaid drugreimbursement benefits on the use of psychotropic agents and acute mental health services by patients with schizophrenia," The New England Journal of 
Medicine 331 (10), September 8, pp. 650- 